\title{
Adiabatic electroweak baryogenesis driven by an axionlike particle
}

\author{
Kwang Sik Jeong, ${ }^{1, *}$ Tae Hyun Jung, ${ }^{2,3, \dagger}$ and Chang Sub Shin $\odot^{2, *}$ \\ ${ }^{1}$ Department of Physics, Pusan National University, Busan 46241, Korea \\ ${ }^{2}$ Center for Theoretical Physics of the Universe, Institute for Basic Science (IBS), Daejeon 34126, Korea \\ ${ }^{3}$ Department of Physics, Florida State University, Tallahassee, FL 32306, USA
}

(Received 14 June 2019; accepted 20 January 2020; published 7 February 2020)

\begin{abstract}
An axionlike particle (ALP) offers a new direction in electroweak baryogenesis because the periodic nature enables it to trigger a strong first-order phase transition insensitively to the decay constant $f$. For $f$ much above $\mathrm{TeV}$, the ALP-induced electroweak phase transition is approximately described by adiabatic processes, distinguishing our scenario for electroweak baryogenesis from the conventional ones. We show that, coupled to the electroweak anomaly, the ALP can naturally realize spontaneous electroweak baryogenesis to solve the matter-antimatter asymmetry problem for $f$ in the range between about $10^{5}$ and $10^{7} \mathrm{GeV}$. In such an ALP window, the $C P$ violation for baryogenesis is totally free from the experimental constraints, especially from the recently improved limit on the electron electric dipole moment. Future searches for ALPs could probe our scenario while revealing the connection between electroweak symmetry breaking and baryogenesis.
\end{abstract}

DOI: 10.1103/PhysRevD.101.035009

\section{INTRODUCTION}

The observed matter-antimatter asymmetry in the Universe is one of the pieces of strong evidence for physics beyond the Standard Model (SM). The rapid sphaleron transitions in the symmetric phase provide a large violation of baryon number, indicating that the baryon asymmetry may have been generated at the electroweak (EW) epoch. This scenario of electroweak baryogenesis (EWBG) looks quite natural and attractive as it invokes SM baryon number violations and is implemented at low temperatures. Viable EWBG is achievable in an extension of the SM in which the electroweak phase transition (EWPT) is sufficiently strong and $C P$ violation is large during the phase transition.

Recently, the ACME II Collaboration improved the limit on the electric dipole moment (EDM) of the electron by about 1 order of magnitude relative to the previous one [1]. Although there would still remain an allowed parameter region in the conventional scenarios of EWBG, the improved limit motivates us to consider an orthogonal direction free from the EDM constraints. Along this direction, the EDM is no longer a hint for EWBG, and other experimental

\footnotetext{
*ksjeong@pusan.ac.kr thjung0720@ibs.re.kr csshin@ibs.re.kr
}

Published by the American Physical Society under the terms of the Creative Commons Attribution 4.0 International license. Further distribution of this work must maintain attribution to the author(s) and the published article's title, journal citation, and DOI. Funded by SCOAP. searches are required to probe the connection between EWPT and baryogenesis. For example, if the electron EDM is suppressed in a model for EWBG due to a cancellation among different contributions, the associated particles may exhibit a specific pattern of masses and couplings [2]. In other models, EWBG may be related with dark matter phenomenology if $C P$ violation for baryogenesis comes from a dark sector [3]. To avoid EDM constraints, one may instead implement EWBG at a much higher temperature than in the conventional scenarios through tachyonic thermal masses [4], for which EWBG yields gravitational waves of much higher frequencies.

In Ref. [5], we have noticed that the axionic extended Higgs sector

$$
V=V\left(|H|^{2}, \sin (\phi / f), \cos (\phi / f)\right)
$$

provides a simple example of EWBG compatible with the electron EDM bound for $f$ above a few TeV if the axionlike particle (ALP) $\phi$ couples to the top quark Yukawa operator. Here, $H$ is the SM Higgs doublet, and $f$ is the ALP decay constant. For $f$ above a few TeV, ALP searches at colliders can give an interesting implication for the origin of the matter-antimatter asymmetry [6-9]. In this scenario, $f$ is restricted to be below about $10 \mathrm{TeV}$ because the bubble wall gets thicker with $f$, suppressing the charge transport in plasma after scattering off a propagating wall. In the context of the standard EWBG, a thick wall seems problematic since baryon asymmetry is mostly produced nonlocally through the diffusion of $C P$ asymmetry in front of the bubble wall and the $B$-violating sphaleron process active in the symmetric phase region away from the wall. 
In this paper, we extend our previous work in Ref. [5] to explore the viability of EWBG at $f$ much above $\mathrm{TeV}$ and its connection to ALP searches. As a source of $C P$ violation, we consider an ALP-dependent EW theta term

$$
\frac{\alpha_{W}}{4 \pi} \Theta_{\mathrm{EW}} W^{a \mu \nu} \tilde{W}_{\mu \nu}^{a},
$$

with

$$
\Theta_{\mathrm{EW}}=\frac{\phi}{f} .
$$

The above coupling can be induced easily, for instance, through loops of extra heavy leptons charged under the ALP shift symmetry, $\phi \rightarrow \phi+$ (constant). It turns out that the standard nonlocal production of baryon asymmetry is highly suppressed, but instead sizable baryon asymmetry can be generated locally as a result of $B$ - and $C P$-violating processes occurring simultaneously near and across the bubble wall. This way, the ALP implements so-called local spontaneous EWBG.

During EWPT, the ALP field changes its value as $\Delta \phi=\mathcal{O}(f)$, and thus the time derivative of $\Theta_{\mathrm{EW}}$ acts as a source for the chemical potential of the Chern-Simons (CS) number at a given spatial point. This leads to the generation of baryon number through the EW anomaly:

$$
\frac{d n_{B}}{d t} \approx \frac{N_{g}}{2} \frac{\Gamma_{\mathrm{sph}}}{T} \frac{d \Theta_{\mathrm{EW}}}{d t}-\Gamma_{B} n_{B},
$$

with $N_{g}=3$ being the number of generations. Here, $\Gamma_{\mathrm{sph}}$ is the sphaleron transition rate per unit volume, and $\Gamma_{B}=$ $\left(13 N_{g} / 4\right) \Gamma_{\text {sph }} / T^{3}$ is the rate of the sphaleron-induced relaxation of baryon asymmetry $[10,11]$.

Local spontaneous EWBG has been studied intensively in the early stage of the development of EWBG [12-17]. However, it was noted that the $C P$-odd scalar in a twoHiggs doublet model cannot give sufficient $C P$ violation for baryogenesis without diffusion effects [14-16]. At that time, there was also a large uncertainty in the baryon asymmetry estimation due to the lack of numerical understanding of how $\Gamma_{\mathrm{sph}}$ changes with the Higgs vacuum expectation value. Furthermore, the realistic bubble wall is not so thick in the usual EWBG models, for which the outof-equilibrium process and charge transport are quite important and most of the baryon asymmetry is produced ahead of the bubble wall. The situation is quite different for EWPT triggered by the ALP because the bubble wall width is much larger than the diffusion length scale in thermal bath. This implies that baryogenesis occurs in the adiabatic limit. The recent lattice calculation of the sphaleron rate shows the dependence on temperature and the Higgs vacuum expectation value [18].

On one hand, an EW theta term varying during EWPT has been studied before, for instance, see Refs. [19,20], but mostly in the context of cold baryogenesis [21]. Those models rely on efficient production of Higgs winding numbers, which could be achieved through a preheating stage with an inflaton coupled the Higgs sector [21], or a delayed first-order phase transition induced by conformal symmetry breaking and subsequent bubble collisions $[22,23]$. Such a violent environment can generate unstable Higgs winding numbers which are large enough to decay through the production of $C P$-violating CS numbers. Another way to induce a time-dependent EW theta term is to consider $C P$ violation from an axion anomalously coupled to a confining hidden gauge group, and its transmission to the SM via messengers [24]. Then, assuming some mechanism for a strong first-order EWPT, EWBG would be realized in the parameter space where the axion slowly rolls and the messenger masses significantly change during EWPT.

In our scenario, the ALP plays the essential role in both EWPT and baryogenesis. We also note that there is no violent out-of-equilibrium process, and all stages of baryogenesis proceed nearly smoothly. This allows us to make a concrete prediction for the baryon asymmetry while establishing an interesting and meaningful relation between EWBG and ALP searches. We find that, feebly coupled to the Higgs sector and EW anomaly, the ALP can naturally solve the puzzle of the matter-antimatter asymmetry in the Universe. Successful baryogenesis is achieved for $f$ below $10^{8} \mathrm{GeV}$, and the model is totally free from the EDM constraints for $f$ much above TeV. The viable window is $f$ between about $10^{5}$ and $10^{7} \mathrm{GeV}$, or equivalently the ALP mass roughly equal to $m_{W}^{2} / f$, i.e., in the $\mathrm{MeV}$ to $\mathrm{GeV}$ range, once the constraints on ALP-Higgs mixing from various experiments are imposed. Our scenario therefore encourages experimental searches for ALPs in the indicated window of parameter space, which would otherwise fall short of strong theoretical interest.

This paper is organized as follows. In Sec. II, we show that a strong first-order phase transition is achievable in the Higgs potential modified by the ALP even in the weakly coupled regime with $f$ much above $\mathrm{TeV}$ and then discuss essential features of the ALP-induced EWPT. In Sec. III, we examine spontaneous EWBG naturally realized by the ALP via its coupling to the EW anomaly. The ALP is subject to various experimental constraints because it mixes with the Higgs boson. We summarize the constraints on the ALP properties in Sec. IV. Section V is devoted to the conclusions.

\section{ELECTROWEAK PHASE TRANSITION}

In this section, we discuss how a strong first-order phase transition is achieved in the Higgs potential modified by the ALP. For an explicit model, we consider the case in which the ALP $\phi$ couples to the mass squared operator of the Higgs field $H$,

$$
V=\lambda|H|^{4}+\mu_{H}^{2}(\theta)|H|^{2}+V_{0}(\theta)+\Delta V_{\mathrm{TH}},
$$

for $\theta \equiv \phi / f$, with 


$$
\begin{aligned}
\mu_{H}^{2}(\theta) & =\mu^{2}-M^{2} \cos (\theta+\alpha), \\
V_{0}(\theta) & =-\Lambda^{4} \cos \theta+\text { constant },
\end{aligned}
$$

under the assumption that $f$ is above the EW scale while other mass parameters $\Lambda, \mu$, and $M$ are around or below the EW scale. Here, $\alpha$ is a constant phase, and $\Delta V_{\mathrm{TH}}$ includes thermal corrections. It is worth noticing that the ALPdependent terms are generated in a controllable way if the ALP shift symmetry is broken solely by nonperturbative effects $[5,25]$.

At a temperature much below $f$ but around or above the EW scale, thermal corrections to $V$ from the SM plasma are still sizable, while those due to the ALP interactions are suppressed by powers of $T / f$. This implies

$$
\Delta V_{\mathrm{TH}} \simeq \Delta V_{\mathrm{TH}}^{\mathrm{SM}}\left(|H|^{2}\right)
$$

where $\Delta V_{\mathrm{TH}}^{\mathrm{SM}}$ includes thermal corrections only from the SM particles. The thermal evolution of the scalar fields is thus described as follows. In phase transition, the most important role is played by the contribution of $\Delta V_{\mathrm{TH}}^{\mathrm{SM}}$ to the Higgs quadratic term. The thermal corrected Higgs mass squared is approximately given by

$$
\mu_{H T}^{2}(\theta) \simeq \mu^{2}-M^{2} \cos (\theta+\alpha)+c_{h} T^{2},
$$

for a positive coefficient $c_{h}$ determined by SM couplings. For sufficiently high temperatures, $\mu_{H T}^{2}$ is positive for all values of $\theta$, making $V$ develop a unique minimum at $(\theta, H)=(0,0)$. For $M^{2}>\mu^{2}$, it is clear that $\mu_{H T}^{2}$ becomes negative in a certain range of $\theta$ if the temperature drops sufficiently, implying that there appears an additional local minimum at $\theta \neq 0$ and $H \neq 0$. The two minima are degenerate when the Universe cools down to $T=T_{c}$, and then a phase transition happens from the symmetric phase to the broken one at a temperature below $T_{c}$. After the phase transition, the scalar fields roll toward the true vacuum.

For the scalar potential (5), $\phi$ and $h$ form two mass eigenstates $\varphi_{L}$ and $\varphi_{H}$ with temperature-dependent masses $m_{L}$ and $m_{H}$, respectively, where $h=\sqrt{2}\left|H^{0}\right|$ denotes the neutral Higgs scalar. For $f$ much above the EW scale, the light scalar $\varphi_{L}$ is mostly the ALP and has a mass, $m_{L} \sim m_{H}^{2} / f$. As can be deduced from such a large mass hierarchy, the field evolution occurs mainly along the direction of the light ALP-like field, and the fluctuation along the direction of the heavy Higgs-like field is quickly damped within the timescale of order $1 / m_{H}$. This feature has been explicitly shown in Appendixes A and B. One can thus examine the structure of phase transition within an effective theory constructed by integrating out the heavy Higgs field via the equation of motion

$$
\left.\frac{\partial V}{\partial h}\right|_{h=\hat{h}(\phi)}=0
$$

where the solution $\hat{h}$ is found to be

$$
\hat{h}(\phi) \simeq\left\{\begin{array}{ll}
0 & \text { for } \mu_{H T}^{2}(\phi) \geq 0 \\
\sqrt{-\frac{1}{\lambda} \mu_{H T}^{2}(\phi)} & \text { for } \mu_{H T}^{2}(\phi)<0
\end{array} .\right.
$$

We note that a more precise solution is obtained if one includes contributions from $\Delta V_{\mathrm{TH}}^{\mathrm{SM}}$ to the Higgs cubic and quartic terms. At a temperature at which $V$ develops two minima, the effect of such contributions is to make the EW minimum deeper and farther from $h=0$, because thermal corrections are Boltzmann suppressed at Higgs field values larger than $T$. Therefore, with the precise solution, one would find that the suppression of sphaleron processes in the broken phase is strengthened as preferred for EWBG. Keeping this in mind, we take Eq. (10) as a good approximation.

It is straightforward to see that the effective potential of the light field reads

$$
V_{\text {eff }}(\phi)=\Lambda^{4}\left(\cos \theta_{0}-\cos \theta\right)-\frac{\lambda}{4}\left(\hat{h}^{4}(\phi)-v_{0}^{4}\right),
$$

where the true minimum at $T=0$ is located at $(\theta, h)=$ $\left(\theta_{0}, v_{0}\right)$. Figure 1 in Ref. [5] illustrates how $V_{\text {eff }}(\phi)$ changes with $T$ and how it is projected from the full potential $V(H, \phi)$. In what follows, we will parametrize the potential in terms of three dimensionless parameters

$$
\alpha, \epsilon \equiv \frac{\sqrt{2 \lambda} \Lambda^{2}}{M^{2}}, \quad r \equiv \frac{\sqrt{2} \Lambda^{2}}{\sqrt{\lambda} v_{0}^{2}},
$$

by imposing the condition $v_{0}=246 \mathrm{GeV}$ and the observed Higgs boson mass to fix $\lambda$ and $\mu$. From the scalar potential, one finds

$$
\sin \theta_{0}=\frac{-\sin \alpha}{\sqrt{1+2 r \epsilon \cos \alpha+r^{2} \epsilon^{2}}},
$$

where the overall sign of $\cos \theta_{0}$ is fixed by the minimization condition.

Let us briefly illustrate the procedure of a first-order phase transition driven by the ALP. At high temperatures, the minimum of $V_{\text {eff }}$ is located at $\theta=0$ because large thermal corrections lead to $\hat{h}(\theta)=0$ in the whole range of $\theta$. The initial position of the ALP at a high temperature is generally displaced from the potential minimum $\theta=0$, but its effect on the phase transition can be safely ignored as long as the ALP potential is developed at a temperature much above the weak scale. For instance, if generated by hidden QCD [5], the ALP mass grows according to 


$$
m_{\phi}(T) \propto\left(\frac{\Lambda_{c}}{T}\right)^{\ell}
$$

as the Universe cools down for $T>\Lambda_{c}$, and it reaches the value equal to $\Lambda^{2} / f$ at $T$ around the hidden confinement scale $\Lambda_{c}$. Here, $\ell=\left(11 N_{c}+N_{f}\right) / 6-2$ and $\Lambda^{4}=m_{N} \Lambda_{c}^{3}$ for a confining $\mathrm{SU}\left(N_{c}\right)$ with $N_{f}$ vectorlike quarks having masses $m_{N} \ll \Lambda_{c}$. The ALP starts coherent oscillations at $T=T_{\text {osc }}$ when the Hubble expansion rate becomes comparable to the ALP mass. Note that $T_{\text {osc }}$ can be well above the weak scale because $\Lambda_{c}$ is higher than $\Lambda$. In the case with $N_{c}=3$ and $N_{f}=1$, oscillation starts at

$$
T_{\mathrm{osc}} \simeq 25 \mathrm{TeV}\left(\frac{\Lambda^{2} / f}{10 \mathrm{MeV}}\right)^{3 / 17}\left(\frac{\Lambda_{c}}{\mathrm{TeV}}\right)^{11 / 17}
$$

and its amplitude is given by

$$
\begin{aligned}
\theta_{\text {osc }}(T) & \simeq \theta_{\text {ini }}\left(\frac{m_{\phi}\left(T_{\text {osc }}\right)}{m_{\phi}(T)}\right)^{1 / 2}\left(\frac{T}{T_{\text {osc }}}\right)^{3 / 2} \\
& =2.7 \times 10^{-6} \theta_{\text {ini }}\left(\frac{T}{\Lambda_{c}}\right)^{3 / 2}\left(\frac{\Lambda_{c}}{\mathrm{TeV}}\right)^{20 / 17},
\end{aligned}
$$

since the ALP number density scales as $T^{3}$ during coherent oscillations. Here, $\theta_{\text {ini }}$ is the initial misalignment angle of the ALP. The above shows that $\theta_{\text {osc }}$ becomes negligibly small at the time of EWPT if the hidden QCD confines at a scale above the weak scale.

When the Universe sufficiently cools down, there appears a region of $\theta$ with nonvanishing $\hat{h}$, which is around $\theta=\epsilon-\alpha$. For $\alpha \neq 0, V_{\text {eff }}$ develops two degenerate minima separated by a barrier at the critical temperature $T=T_{c}$. Then, EW bubbles of the broken phase are nucleated and expand. The EW minimum gets deeper than the symmetric one as $T$ decreases, and the bubble nucleation rate per unit volume exceeds the Hubble expansion rate at $T=T_{n}$. Finally, the potential barrier between two minima disappears at $T=T_{2}$,

$$
T_{2}=\sqrt{1-\frac{r}{\epsilon}\left(\cos \left(\theta_{0}+\alpha\right)-\cos \alpha\right)} T_{c}^{\mathrm{SM}},
$$

where $T_{c}^{\mathrm{SM}}=\sqrt{\lambda / c_{h}} v_{0} \simeq 150 \mathrm{GeV}$ is the critical temperature for the SM Higgs sector. The phase transition is thus first order and is strong if $v_{c} / T_{c}>1$ with $v_{c}$ being the Higgs vacuum expectation value at $T_{c}$. Interestingly, a strong first-order phase transition is achievable even for $f$ much above the EW scale, i.e., in the weakly coupled limit, which distinguishes our model from the conventional approaches.

Figure 1 illustrates how EWPT takes place depending on $\alpha$ and $\epsilon$ for the case with $\Lambda=130 \mathrm{GeV}$, which corresponds to $r \simeq 1.1$. A first-order phase transition is achieved in the white and red regions, and it is strong in the white region.

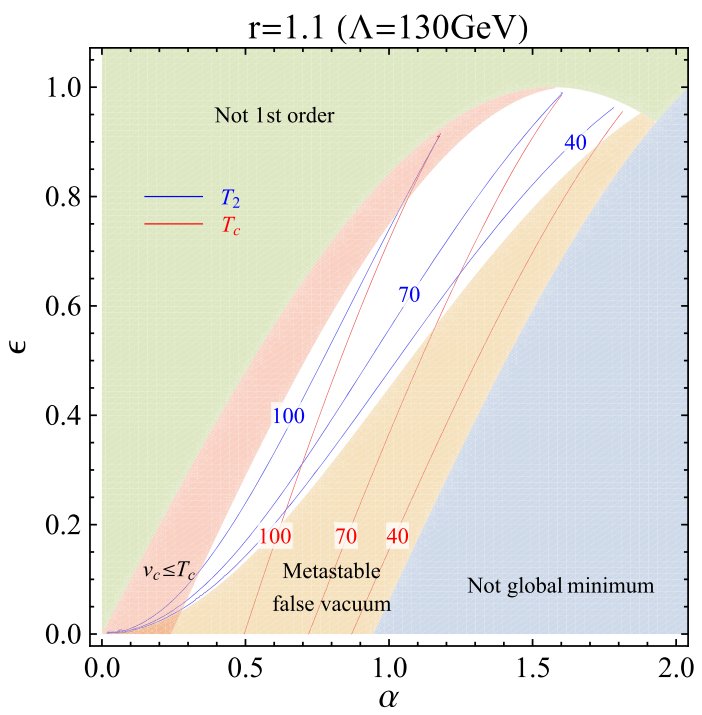

FIG. 1. EWPT in the Higgs sector modified by the ALP for $r=1.1$. A strong first-order phase transition occurs in the white region, insensitively to the value of $f$. The red and blue lines show the critical and bubble disappearing temperatures in the $\mathrm{GeV}$ unit, respectively.

In the region of a first-order phase transition, we also show the constant contours of $T_{2}$ by blue lines and those of $T_{c}$ by red lines. The blue region leads to an EW minimum higher than the symmetric minimum, while the orange region is excluded because the vacuum transition rate to the EW minimum is highly suppressed for $f$ above $\mathrm{TeV}$ due to a barrier remaining at $T=0$. In the green region, the phase transition is not first order as in the SM. We note that the indicated lines and regions in the figure change only slightly with $f$ because the potential for fixed $\theta$ does not rely on it at the tree level. Let us shortly discuss the generic behavior for a different value of $r \propto \Lambda^{2}$. If one increases $r$, a strong first-order phase transition occurs at smaller values of $\epsilon$ for a given $\alpha$ because it requires sizable $\sin \theta_{0}$. Thus, the white region in Fig. 1 moves to the bottom right. In the opposite case with a smaller value of $r$, it moves to the top left. The remaining qualitative behaviors are the same as before.

Let us examine the phase transition in more detail. The bubble nucleation rate is given by $T^{4} e^{-S_{3} / T}$, where $S_{3}$ is the Euclidean action of an $O(3)$ symmetric critical bubble. For $f$ above $\mathrm{TeV}$, the contribution to $S_{3}$ from Higgs kinetic terms is highly suppressed, and tunneling occurs dominantly along the ALP direction. Interestingly, combined with the insensitivity of the scalar potential to $f$ for given $\theta$, this leads to the approximate scaling laws

$$
S_{3} \propto f^{3}, \quad R_{c} \propto f,
$$

where $R_{c}$ is the radius of the critical bubble. See Appendix A for the details. At temperatures around $T_{n}$, one can thus take an approximation, 


$$
\frac{S_{3}}{T} \propto\left(T-T_{2}\right)^{n} f^{3},
$$

for a positive constant $n$ of order unity, where we have used that $S_{3}=0$ at $T=T_{2}$ because there is no potential barrier. Thus, there are characteristic features specific to our scenario. One is that the bubble nucleation temperature, which is determined by $S_{3} / T \approx 130$, is close to the barrier disappearing temperature

$$
T_{n} \sim T_{2},
$$

where the difference between the two is suppressed by a factor of $f^{-3 / n}$. Another distinctive feature is that bubbles are formed with a thick wall roughly proportional to $f$, and the phase transition proceeds rather smoothly with nucleation of bubbles. This implies that the phase transition is approximately adiabatic during baryogenesis, and diffusion through the bubble wall is not efficient for large $f$.

It follows from the scaling behavior of $S_{3}$ that the duration of phase transition decreases with $f$ as

$$
\Delta t_{\mathrm{PT}} \simeq \frac{6}{-d\left(S_{3} / T\right) /\left.d\right|_{T_{n}}} \propto \frac{1}{f^{3 / n}} .
$$

For $r$ of order unity, a numerical analysis shows

$$
\Delta t_{\mathrm{PT}} \sim \frac{10^{-2}}{H}\left(\frac{1 \mathrm{TeV}}{f}\right)^{3 / n},
$$

with $1 \lesssim n \lesssim 2$, in the parameter region of a first-order phase transition. Here, $H$ is the Hubble expansion rate at $T=T_{n}$.

On the other hand, the wall width in the rest frame of a bubble wall can be regarded as the size of the critical bubble, and it is given by

$$
L_{w} \sim \sqrt{\frac{\Delta \Phi_{c}^{2}}{\Delta V_{c}}} \sim \frac{f}{\Lambda^{2}},
$$

where $\Delta \Phi_{c}$ is the field variation during tunneling and $\Delta V_{c} \sim \Lambda^{4}\left(\Delta \Phi_{c} / f\right)^{2}$ is the height of the potential barrier. One can see that the bubble wall is thick, $L_{w} \gtrsim 100 / T_{n}$, for $f$ above $10^{4} \mathrm{GeV}$ and $T_{n}$ around $50 \mathrm{GeV}$. This corresponds to the adiabatic regime, in which nonthermal enhancement of baryon production is expected neither from particle diffusion $[15,16]$ nor the classical dynamics of fast Higgs quenching [22].

If $f$ is even larger to give $L_{w}>v_{w} \Delta t_{\mathrm{PT}}$ with $v_{w}$ being the wall velocity, the phase transition proceeds via bubble nucleation but without substantial expansion of bubbles. This happens when $f \gtrsim 10^{6} \mathrm{GeV}$ for $n=1$ and $f \gtrsim$ $10^{8} \mathrm{GeV}$ for $n=2$, where we have taken $v_{w} \sim 0.1$. It is also important to note that bubble nucleation is followed by rolling of the ALP toward the true minimum of the potential after tunneling. The phase transition looks smooth for $T_{n}$ close to $T_{2}$, but it is definitely distinguishable from a second-order one because its large mass makes the ALP evolve much more quickly compared to the cooling rate of the Universe.

For a final remark in this section, we note that a singlet scalar can play a similar role in EWPT as the ALP in our model under certain conditions on its couplings. Let us consider an extension with a real scalar $s$ :

$$
V=\lambda|H|^{4}+\mu_{H}^{2}(s)|H|^{2}+V_{0}(s) .
$$

For the scalar feebly coupled to $H$, a first-order phase transition is still possible if $\mu_{H}^{2}$ is negative in a finite range of $s$ and $V_{0}$ is bounded from below and has a single minimum lying in the region where $\mu_{H}^{2}$ is negative. Here, $V_{0}$ should not be too steep around the minimum so that the high temperature potential can properly develop symmetric and EW minima separated by a barrier. For a simple example, we consider

$$
\begin{aligned}
\mu_{H}^{2} & =-\mu^{2}+\lambda_{h s}\left(s-\mu_{s}\right)^{2}, \\
V_{0} & =\sum_{n=1}^{4} \lambda_{n} \mu_{s}^{4-n} s^{n},
\end{aligned}
$$

for a positive coupling $\lambda_{h s} \ll 1$. Under the assumption for simplicity that $V_{0}$ has a single minimum at $s=0$, the conditions for a first-order phase transition read

$$
\begin{aligned}
& 0<-\mu_{H}^{2}(s=0) \sim v_{0}^{2}, \\
& V_{0}\left(s=\mu_{s}\right)-V_{0}(s=0)<\frac{\mu^{4}}{4 \lambda} \sim v_{0}^{4},
\end{aligned}
$$

implying $\lambda_{h s} \sim\left(v_{0} / \mu_{s}\right)^{2}$ and $\sum_{n} \lambda_{n} \lesssim\left(v_{0} / \mu_{s}\right)^{4}$. Such a hierarchical structure of singlet couplings would indicate some underlying symmetry. An ALP is therefore a natural candidate because its couplings are controlled by the associated shift symmetry. In this case, $\mu_{s}$ corresponds to the ALP decay constant. An important feature of the ALP extension is that one can control separately the strength of couplings and the strength of EWPT since the latter is insensitive to the decay constant. Furthermore, the periodic nature allows us to avoid the instability problem of the scalar potential independently of the details of the model.

\section{BARYOGENESIS}

Coupled to the Higgs mass squared operator, the ALP makes EWPT strongly first order in a wide range of parameter space including the weakly coupled regime with large $f$. Furthermore, its coupling to the EW anomaly provides a sizable chemical potential for the CS number during phase transition. As a result, the ALP naturally 

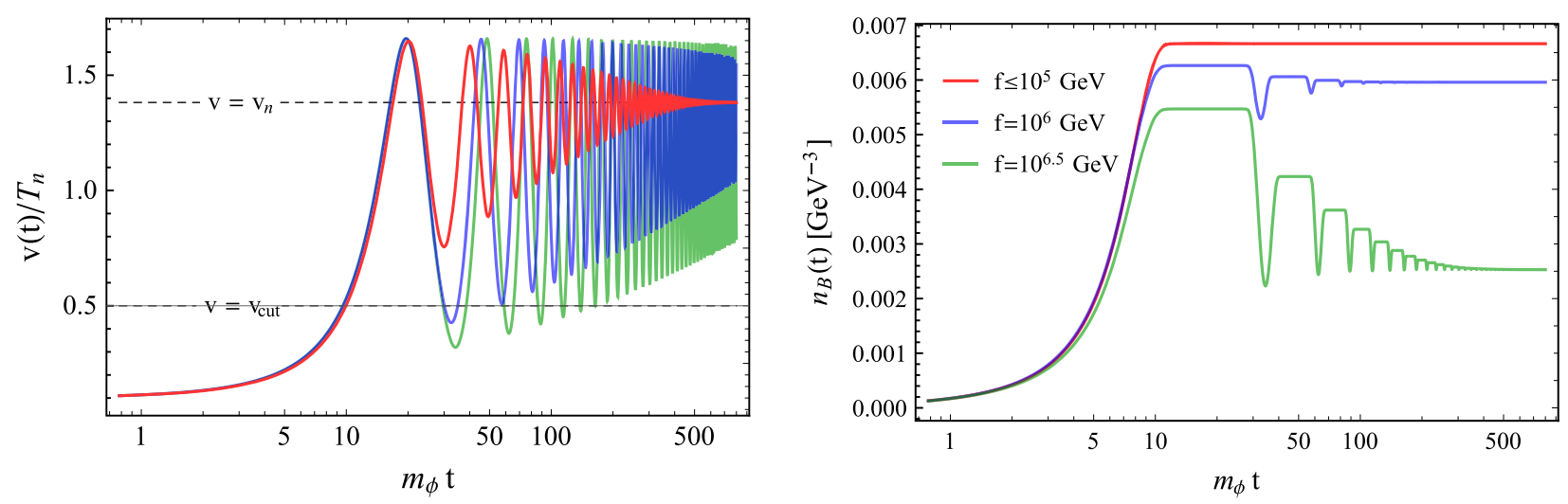

FIG. 2. Evolutions of the Higgs background field value (left) and baryon number density (right) in the case with $\alpha=1.4, \epsilon=0.95$, and $r=1.1$ for different values of $f$ as indicated in the figure. Here, $m_{\phi}$ is the ALP mass. The Higgs background field value oscillates about the potential minimum $v=v_{n}$, and sphalerons are active for $v$ smaller than $v_{\text {cut }}$. The right panel shows that baryon asymmetry is efficiently produced during the first falling toward $v=v_{n}$, and then it is washed out by later oscillations if the oscillation reaches the region with $v \lesssim v_{\text {cut }}$.

realizes spontaneous EWBG to solve the matter-antimatter asymmetry problem.

A distinctive feature of ALP-induced EWPT is that it is approximately adiabatic for $f$ above $10 \mathrm{TeV}$. Then, a thick bubble wall makes diffusion effects inefficient, implying that nonlocal baryon production can be neglected for large $f$, where the wall gets thicker proportional to $f$ as discussed in Sec. II. The ALP induces local baryon production by providing a CS chemical potential. Another intriguing feature, which will be discussed below, is that baryogenesis proceeds almost isothermally if $T_{n}$ is above about $30 \mathrm{GeV}$. This makes the situation simple to analyze.

Let us now examine the ALP evolution during phase transition. The ALP undergoes an underdamped oscillation inside bubbles following the equation of motion

$$
\ddot{\phi}+\left(3 H+\Upsilon_{\phi}^{\mathrm{eff}}\right) \dot{\phi}+\frac{d V_{\mathrm{eff}}}{d \phi}=0,
$$

where the dot denotes a derivative with respect to time $t$ and $\Upsilon_{\phi}^{\text {eff }}$ is the effective energy transfer rate from the ALP field to other particles and bubbles. For $f$ above about $10 \mathrm{TeV}$, the typical timescale characterizing the dynamics of SM thermal plasma, which is roughly $1 /\left(\alpha_{s}^{2} T_{n}\right)$, is much shorter than the timescale of the field variations approximately $1 / m_{\phi}$. The baryon asymmetry can then be numerically calculated by solving Eqs. (4) and (27) as shown in Fig. 2.

On the other hand, it is also possible to analytically understand how baryogenesis proceeds. The solution to Eq. (4) can be written in the integral form:

$$
n_{B}(t)=\int_{0}^{t} d t^{\prime} \frac{3 \Gamma_{\mathrm{sph}}}{2 T} \dot{\theta} \operatorname{Exp}\left[-\int_{t^{\prime}}^{t} d t^{\prime \prime} \frac{39 \Gamma_{\mathrm{sph}}}{4 T^{3}}\right] .
$$

It is convenient to separate the ALP evolution into two parts, the first falling toward the potential minimum and later oscillations. It is during the first falling that baryon asymmetry is efficiently created while passing the region with small $v$ where sphalerons are active. The relaxation of baryon asymmetry is negligible at this stage. On the contrary, the effect of later oscillations is only to wash out the baryon asymmetry because a cancellation occurs between baryon and antibaryon numbers produced by the CS chemical potential at each oscillation. Using the fact that the first falling and later oscillations of the ALP play different roles in baryogenesis, one can reduce the solution of the integral form to ${ }^{1}$

$$
n_{B}=n_{0} e^{-K_{\phi}},
$$

in which $n_{0}$ is determined by the baryon asymmetry produced during the first falling

$$
n_{0} \simeq 27 \alpha_{W}^{5} T_{n}^{3} \Delta \theta,
$$

and the exponential factor represents the washout during oscillations,

$$
K_{\phi} \simeq \frac{351 \alpha_{W}^{5} T_{n}}{2}\left(\Delta t_{0}+2 \sum_{i=1}^{N_{\text {osc }}} \Delta t_{i}\right)
$$

Here, $\Delta t_{0}$ is the duration of the first falling, and $N_{\text {osc }}$ counts the number of oscillations such that sphalerons are unsuppressed during $t_{i}-\Delta t_{i}<t<t_{i}+\Delta t_{i}$ around the peak of the $i$ th oscillation. The interval of $\theta$ where $\hat{h}(\theta)$ is smaller than $v_{\text {cut }}$ during the first falling is estimated to be

\footnotetext{
${ }^{1}$ More generally, the ALP coupling to the EW anomaly reads $\Theta_{\mathrm{EW}}=N_{\mathrm{EW}} \phi / f$, where an integer $N_{\mathrm{EW}}$ is model dependent. We shall take $N_{\mathrm{EW}}=1$ throughout the paper, but one can consider a different value to enhance the baryon asymmetry.
} 


$$
\Delta \theta \simeq \frac{\epsilon}{r \sin \alpha} \frac{v_{\mathrm{cut}}^{2}}{v_{0}^{2}}
$$

where we have used that the phaleron rate reads $\Gamma_{\text {sph }} \approx 18 \alpha_{W}^{5} T^{4}$ if the Higgs background field value is smaller than $v_{\text {cut }} \simeq 0.5 T$, and it is exponentially suppressed otherwise [18].

The correct baryon asymmetry is obtained if the ALP evolution does not cause strong washout. Let us examine the conditions for this. The temperature is kept near $T_{n}$ during all stages of baryogenesis, and thus one needs

$$
\frac{v_{n}}{T_{n}} \gtrsim 1,
$$

which is slightly weaker than the condition for a strong first-order phase transition illustrated in Fig. 1 . Here, $v_{n}$ is the Higgs vacuum expectation value at $T_{n}$. In addition, the friction term should quickly reduce the ALP oscillation amplitude so that the Higgs background field value

$$
v(t) \equiv \hat{h}(\theta(t))
$$

is smaller than $v_{\text {cut }}$ afterward. This requires

$$
f<10^{8} \mathrm{GeV},
$$

because the ALP dissipates energy into the background plasma through interactions with SM particles induced by scalar mixing. For $f$ in the opposite region, bubbles do not expand substantially during the phase transition, and so the friction comes dominantly from thermal dissipation from the coupling to the top quarks through the ALP-Higgs mixing [26,27],

$$
\Upsilon_{\phi}^{\text {eff }} \sim\left\{\begin{array}{ll}
y_{t}^{2} \Gamma_{\mathrm{th}} \sin ^{2} \delta(t) & \text { for } y_{t} v(t) \lesssim \Gamma_{\mathrm{th}} \\
y_{t}^{2} \frac{\left(y_{t} v(t)\right)^{2}}{\Gamma_{\mathrm{th}}} \sin ^{2} \delta(t) & \text { for } y_{t} v(t) \gtrsim \Gamma_{\mathrm{th}}
\end{array},\right.
$$

with the thermal width $\Gamma_{\text {th }} \simeq 0.1 T$ determined by the top quark Yukawa coupling $y_{t}$ and the QCD gauge coupling. Here, the ALP-Higgs mixing angle $\delta$ changes with time as $v(t)$ does. See Appendix B for more discussion on the evolution of Higgs and ALP fields including other sources of dissipation. In the numerical analysis, we neglect the contribution of $\mathcal{O}\left(g_{i}^{2}\right)$ in the denominator. During the time when the ALP passes the region with $v$ larger than $T_{n}$, the top quark decouples from thermal equilibrium, and dissipation gets suppressed by the Yukawa couplings of other light fermions. For $f$ around and above $10^{8} \mathrm{GeV}$, the exponent $K_{\phi}$ is larger than order unity and scales roughly with $1 / f^{2}$, and thus it corresponds to the strong washout regime. Note that the ALP decay is highly suppressed, but occurs well before nucleosynthesis for $f$ below $10^{8} \mathrm{GeV}$.
The estimation of baryon asymmetry also requires knowing how much temperature changes during baryogenesis. After the phase transition, the ALP settles down to the potential minimum, and the Universe heats up to the temperature $T=T_{\text {reh }}$ with $T_{\text {reh }}$ determined by

$$
\left(\frac{T_{\text {reh }}}{T_{n}}\right)^{4} \simeq 1+0.1\left(\frac{\Delta V}{(80 \mathrm{GeV})^{4}}\right)\left(\frac{60 \mathrm{GeV}}{T_{n}}\right)^{4},
$$

taking $g_{*}=100$. Here, $\Delta V$ is the difference of vacuum energy densities of the symmetric and broken phases at $T_{\text {reh }}$, and so it is a fraction of the former, $V_{\text {eff }}(\theta=0)$. The above relation indicates that extra entropy production is negligible, i.e.,

$$
T_{\text {reh }} \simeq T_{n},
$$

unless $T_{n}$ is lower than about $30 \mathrm{GeV}$.

Finally, taking into account the effects discussed so far, we find the baryon-to-entropy ratio to be

$$
\frac{n_{B}}{S} \simeq \frac{1}{\Delta} \frac{2.6 \epsilon \times 10^{-10}}{r \sin \alpha}\left(\frac{T_{n}}{60 \mathrm{GeV}}\right)^{2},
$$

where we have taken $g_{*}=100$ and $\Delta \simeq\left(T_{\text {reh }} / T_{n}\right)^{3} e^{K_{\phi}}$ describes the dilution of baryon number. The above relation shows that spontaneous EWBG induced by the ALP can account for the observed baryon asymmetry, $n_{B} / s \simeq$ $8.6 \times 10^{-11}$, if $\Delta$ lies in the range

$$
1 \leq \Delta \lesssim 10
$$

which is the case for $T_{n}$ above about $30 \mathrm{GeV}$ and $f$ below $10^{8} \mathrm{GeV}$. The dilution factor exponentially increases for larger $f$ or in the region with $v_{n} / T_{n}<1$. It should be noted that $T_{n}$ is close to $T_{2}$, and the dependence of $f$ of the baryon asymmetry comes in through the washout factor. Thus, in the small washout regime with $\Delta$ below about 10 , the baryon asymmetry becomes not very dependent on $f$.

Let us show the evolutions of relevant physical quantities on figures. In the left panel of Fig. 2, the curves show how the Higgs background field value evolves for $f$ between $10^{6}$ and $10^{7} \mathrm{GeV}$ in the case with

$$
\alpha=1.4, \quad \epsilon=0.95, \quad r=1.1,
$$

for which $T_{2} \simeq 84 \mathrm{GeV}$. The scalar potential is asymmetric about the minimum $v=v_{n}$ for nonzero $\alpha$ and $\epsilon$, and sphalerons are active only in the region below the lower horizontal dashed line, where $v<v_{\text {cut }}$. One can see that the number of relevant oscillations decreases with $f$. The right panel shows the evolution of the baryon number. The baryon number is produced at the first falling, and then is washed out if later oscillations pass the region of rapid sphaleron transitions. 


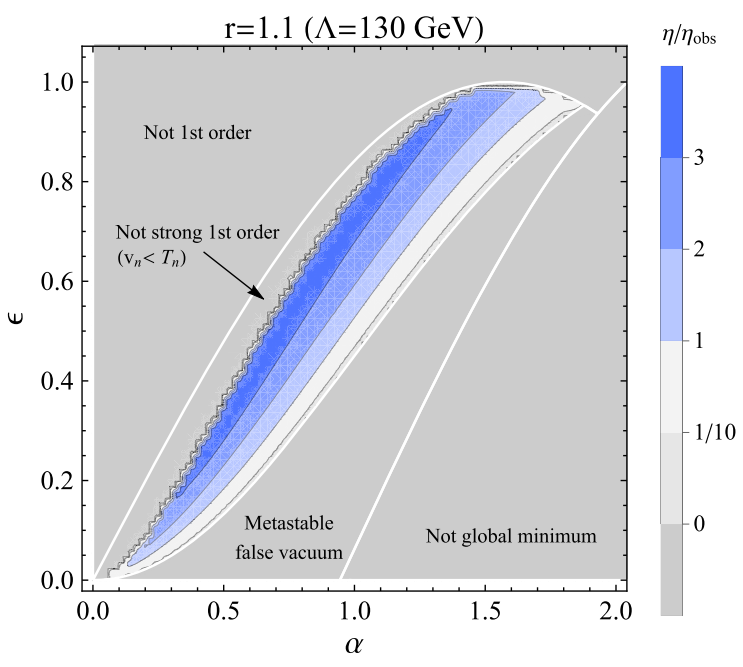

FIG. 3. Spontaneous EWBG realized by the ALP in the case with $r=1.1$ and $f=10^{6} \mathrm{GeV}$. The gradient represents the relic baryon number density normalized by the observed value. The correct baryon asymmetry is obtained in the blue shaded region for an appropriate dilution factor. Sizable dilution requires $f$ above $10^{7} \mathrm{GeV}$, below which the baryon asymmetry becomes insensitive to $f$.

We close this section by examining the viable region of parameter space for the case with

$$
r=1.1, \quad f=10^{6} \mathrm{GeV} .
$$

The blue shaded region in Fig. 3 leads to the correct baryon asymmetry, and the color gradient represents the required value of dilution factor $\Delta$. Note that sizable dilution is obtained for $f$ above $10^{7} \mathrm{GeV}$. Here, we have used that $T_{n}$ is close to $T_{2}$, which does not depend on $f$, and that the region for a first-order phase transition with $v_{n} / T_{n}>1$ does not change much with $f$ because $V_{\text {eff }}(\theta)$ relies on $f$ only via small radiative corrections. This also indicates that the viable region would remain almost the same if one considers smaller $f$.

\section{EXPERIMENTAL CONSTRAINTS}

In this section, we summarize experimental constraints on the ALP. The coupling to the Higgs mass squared operator makes the ALP mix with the Higgs boson, and thus there are various constraints depending on the mixing angle

$$
\sin \delta \simeq \frac{r^{2} \sin \theta_{0}}{2} \times \frac{v_{0}}{f}
$$

and its mass

$$
m_{\phi} \simeq \sqrt{\frac{r^{2}\left(\sin \alpha+r^{3} \epsilon \sin ^{3} \theta_{0}\right)}{4 \sin \left(\theta_{0}+\alpha\right)}} \times \frac{v_{0} m_{h}}{f},
$$

where $m_{h} \simeq 125 \mathrm{GeV}$ is the Higgs boson mass. First, the ALP is subject to the bound on the EDM because its coupling to EW anomaly violates $C P$ symmetry in the presence of mixing with the Higgs boson. The electron EDM is radiatively generated as [28]

$$
\begin{aligned}
d_{e} & \simeq \frac{8 e^{3}}{(4 \pi)^{4}} \frac{m_{e}}{v_{0}} \frac{\sin \delta}{f} \ln \left(\frac{m_{h}}{m_{\phi}}\right) \\
& \sim 10^{-34} e \mathrm{~cm} \times\left(\frac{10^{6} \mathrm{GeV}}{f}\right)^{2},
\end{aligned}
$$

where $m_{e}$ is the electron mass. If $f$ is larger than about $5 \mathrm{TeV}$, the above contribution is below the latest experimental bound from ACME II in the region of parameter space for a strong first-order phase transition. One may naively expect that such a large $f$ would also suppress $C P$-violating effects on baryogenesis since the ALP is responsible for the CS chemical potential. However, as shown in Sec. III, the baryon asymmetry is generated depending on how rapidly $\phi / f$ changes during EWPT. The ALP excursion $\Delta \phi$ is of the order of $\alpha f$ during EWPT, and thus spontaneous EWBG can work at $f$ much above $\mathrm{TeV}$ while being free from the EDM constraints. On the other hand, other EWBG scenarios generally suffer from the EDM constraints because the Higgs sector is modified by a singlet scalar significantly coupled to it to induce a strong first-order phase transition. For instance, another simple candidate for a time-dependent EW theta would be $\Theta_{\mathrm{EW}}=$ $|H|^{2} / \Lambda_{\text {cut }}^{2}$, where $\Lambda_{\text {cut }}$ is the cutoff scale of the effective coupling. Then, baryon asymmetry is produced during phase transition according to $n_{B} \propto v_{\text {cut }}^{2} / \Lambda_{\text {cut }}^{2}$ in the adiabatic limit. The correct baryon asymmetry requires $\Lambda_{\text {cut }}$ lower than $0.5 \mathrm{TeV}$ if the phase transition occurs around the EW scale. However, the latest bound on electron EDM from ACME II excludes $\Lambda_{\text {cut }}$ below about $6 \times 10^{5} \mathrm{GeV}$.

Our scenario solves the matter-antimatter asymmetry problem while avoiding the electron EDM bound in the weakly coupled regime with $f$ between about $5 \mathrm{TeV}$ and $10^{8} \mathrm{GeV}$. This corresponds to the ALP mass in the range between sub-MeV and $5 \mathrm{GeV}$, for which stringent constraints come from rare meson decays and also from beamdump ALP searches [29]. In addition, if lighter than about $20 \mathrm{MeV}$, which is roughly the supernova temperature, ALPs can be produced in supernovae. Supernova cooling is accelerated if the produced ALPs efficiently escape from it, implying that the ALP-Higgs mixing should lie in a certain range to avoid conflict with the observation.

Let us describe the experimental constraints from meson decays in more detail. If the ALP has a mass in the range between $2 m_{\mu}$ and $m_{B}-m_{K}$, where $m_{i}$ denotes the mass of the indicated particle, the mixing should be suppressed to be consistent with the limit on the decay rate for $B \rightarrow K \phi \rightarrow$ $K \mu^{+} \mu^{-}$obtained at Belle and LHCb [30-34]. The electron channel, $B \rightarrow K \phi \rightarrow K e^{+} e^{-}$, gives weaker constraint than 


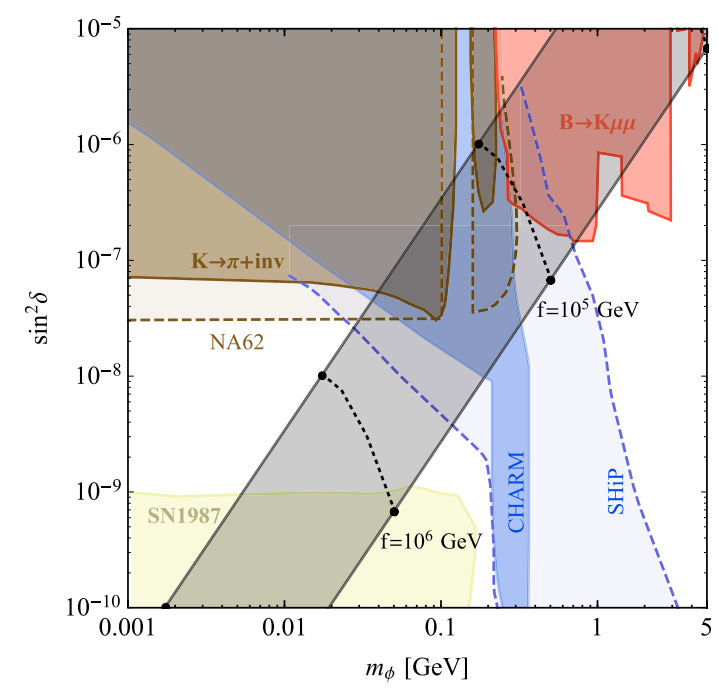

FIG. 4. Experimental constraints on the ALP properties from rare meson decays, supernova cooling, and beam-dump ALP searches [29]. Here, $\delta$ is the ALP-Higgs mixing angle, and the ALP is assumed not to decay into hidden particles. The light blue and purple regions will be reached in future experiments. The gray band shows the relation between the ALP mass and mixing for $r=1.1$ by taking $0.1 \leq \alpha \leq 1.4$ and $0.14 \leq \epsilon \leq 0.8$. The black dotted lines on the band are the contours of $f$.

the muonic one. On the other hand, in the case with $m_{\phi}<$ $m_{K}-m_{\phi}$, the mixing is constrained mainly by rare $K$ meson decays. Especially, if $m_{\phi}<2 m_{\mu}$, the ALP is subject to a stringent bound imposed by the searches for invisible $K$ meson decays at BNL E787 and E949 experiments [35,36].

In Fig. 4, we summarize the current experimental constraints on the ALP properties for $1 \mathrm{MeV} \lesssim m_{\phi} \lesssim 5 \mathrm{GeV}$. The dark purple and cyan regions are excluded by rare $K$ and $B$ meson decays, respectively. The sky blue region leads to too rapid supernova cooling, while the red region is excluded by the beam-dump ALP searches at CHARM [37]. One can see that the viable window is

$$
10^{-9} \lesssim \sin ^{2} \delta \lesssim 3 \times 10^{-7}
$$

for $1 \mathrm{MeV} \lesssim m_{\phi} \lesssim 0.2 \mathrm{GeV}$, while it is

$$
\operatorname{Br}\left(\phi \rightarrow \mu^{+} \mu^{-}\right) \times \sin ^{2} \delta \lesssim 6 \times 10^{-7},
$$

for $0.3 \mathrm{GeV} \lesssim m_{\phi} \lesssim 5 \mathrm{GeV}$, where $\operatorname{Br}\left(\phi \rightarrow \mu^{+} \mu^{-}\right)$is the branching ratio for the ALP decay into a muon pair. Here, we have assumed that the ALP does not decay into hidden sector particles. If allowed, the constraint from $B$ meson decays will be weakened. In our scenario, the approximate relation

$$
m_{\phi} \sim m_{h} \sin \delta
$$

holds between the ALP mass and mixing angle. The gray band shows such a relation for $r=1.1$ in the parameter space, $0.1 \leq \alpha \leq 1.4$ and $0.14 \leq \epsilon \leq 0.8$. Thus, a viable region appears for $m_{\phi}$ in the $\mathrm{MeV}$ to $\mathrm{GeV}$ scale, or equivalently $f$ in the range between about $10^{5}$ and $10^{7} \mathrm{GeV}$. We also plot the constant contours of $f$ on the band by black dotted lines. It is interesting to note that the light blue and brown regions will be probed by experiments at SHiP [38] and NA62, respectively.

\section{CONCLUSIONS}

In this paper, we have shown that an ALP provides a simple and natural framework for EWBG in a wide range of $f$ owing to its periodic nature. In particular, for $f$ much above $\mathrm{TeV}$, it offers a new direction in which the EDM and the LHC are no longer a probe of EWBG. Instead, ALP searches would reveal the interesting connection between EW symmetry breaking and baryogenesis established in our scenario. A part of the parameter space is already constrained by the existing results from ALP searches.

The ALP triggers a strong first-order phase transition insensitively to the value of $f$ and leads to the adiabatic regime at $f$ above $10 \mathrm{TeV}$, where thick bubble walls prevent nonlocal baryon production from particle diffusion. Nonetheless, coupled to the EW anomaly, the ALP can naturally realize local spontaneous EWBG to solve the matterantimatter asymmetry problem for $f$ below $10^{8} \mathrm{GeV}$ while avoiding strong washout. Interestingly, the phase transition occurs smoothly because the bubble nucleation temperature is close to the bubble disappearing temperature, and baryogenesis proceeds almost isothermally.

Using that the ALP-Higgs mixing is constrained by various experiments, we find the viable window to be $f$ from about $10^{5}$ to $10^{7} \mathrm{GeV}$, which corresponds to ALP mass in the $\mathrm{MeV}$ to $\mathrm{GeV}$ scale. In such a weakly coupled regime, our scenario is completely safe not only from the EDM constraints, especially from the bound on the electron EDM recently improved by ACME II, but also from Higgs precision measurements. These are the features distinguishable from the conventional scenarios of EWBG. The indicated ALP window, which has suffered from the lack of strong theoretical interest, could be probed in future beam-dump experiments such as SHiP.

\section{ACKNOWLEDGMENTS}

T. H. J. thanks Géraldine Servant and Eibun Senaha for useful discussions. This work was supported by IBS under the Project code IBS-R018-D1 (T. H. J. and C. S. S.) and by the National Research Foundation of Korea (NRF) grant funded by the Korea government (MSIP) (Grant No. NRF2018R1C1B6006061) (K. S. J.). T. H. J. is also supported by the US Department of Energy grant DE- SC0010102 and Prof. Kohsaku Tobioka's startup fund at Florida State University (Project id: 084011- 550- 042584). 


\section{APPENDIX A: BOUNCE SOLUTION}

In this Appendix, we analyze the structure of the bounce solution in more detail and discuss how the tunneling proceeds. The Higgs and ALP fields are fixed at the symmetric false vacuum, $(\phi, h)=(0,0)$, at high temperatures. As temperature drops, the potential develops a new minimum at $(\phi, h) \neq(0,0)$, and the false vacuum can decay via nucleation of critical bubbles of the broken phase if it has a higher free energy than the EW vacuum. The nucleation rate per unit volume is

$$
\Gamma \propto T^{4} e^{-S_{3} / T},
$$

where the Euclidean action for the bounce is given by

$$
S_{3}=4 \pi \int_{0}^{\infty} d r r^{2}\left[\frac{1}{2}\left(\frac{d h}{d r}\right)^{2}+\frac{1}{2}\left(\frac{d \phi}{d r}\right)^{2}+V\right],
$$

for the scalar potential $V$ at a temperature $T$. Here, we have set $V=0$ at the symmetric vacuum. The bounce field configuration can be found from

$$
\frac{d^{2} \varphi}{d r^{2}}+\frac{2}{r} \frac{d \varphi}{d r}=\partial_{\varphi} V
$$

under the boundary conditions, $d \varphi / d r=0$ at $r=0$ and $\varphi=0$ at $r=\infty$, where $\varphi=\{\phi, h\}$.

It is usually the case that one needs to take numerical calculations to find the bounce solution. However, in our scenario, a large $f$ allows an analytic approach because bubble nucleation occurs mostly along the light field direction and the potential is insensitive to $f$ for a given $\theta$, where $\theta=\phi / f$. This feature becomes transparent when the action is written in the form

$$
S_{3}=4 \pi f^{3} \int_{0}^{\infty} d x x^{2}\left[\frac{h^{\prime 2}}{2 f^{2}}+\frac{\theta^{\prime 2}}{2}+V(h, \theta)\right],
$$

where $x \equiv r / f$ and the prime is the derivative with respect to $x$. The equations of motion then read

$$
\begin{aligned}
\frac{1}{f^{2}}\left(h^{\prime \prime}+\frac{2}{x} h^{\prime}\right) & =\partial_{h} V, \\
\theta^{\prime \prime}+\frac{2}{x} \theta^{\prime} & =\partial_{\theta} V .
\end{aligned}
$$

For the potential (5), field variations over the critical bubble are roughly given by $\Delta h \sim \Lambda$ and $\Delta \theta \sim \alpha$. Using the equation of motion for $\theta$, one can estimate the size of the bubble to be $\Delta x \sim 1 / \Lambda^{2}$ because the insensitivity of $V(h, \theta)$ to $f$ implies

$$
\Delta \theta \partial_{\theta} V \sim \alpha \Lambda^{4} .
$$

Combined with these relations, the equation of motion for $h$ leads to

$$
\Delta h \partial_{h} V \sim\left(\frac{\Lambda}{f}\right)^{2} \Lambda^{4}
$$

Therefore, for $\Lambda \ll f$, the Higgs trajectory for the bounce is effectively fixed by $\partial_{h} V=0$, making the ALP feel a potential along it. This justifies why the tunneling can be examined within the effective theory of the light ALP constructed by integrating out the heavy field $h$,

$$
S_{3} \simeq 4 \pi f^{3} \int_{0}^{\infty} d x x^{2}\left[\frac{\theta^{\prime 2}}{2}+V_{\text {eff }}(\theta)\right],
$$

where $V_{\text {eff }}(\theta)=V(\hat{h}(\theta), \theta)$ for the Higgs field value $h=$ $\hat{h}(\theta)$ satisfying $\partial_{h} V=0$. The bounce solution is obtained from

$$
\theta^{\prime \prime}+\frac{2}{x} \theta^{\prime}=\partial_{\theta} V_{\mathrm{eff}}
$$

under the boundary conditions, $\theta^{\prime}=0$ at $x=0$ and $\theta=0$ at $x=\infty$. Note that the equation of motion is independent of $f$, implying $S_{3} \propto f^{3}$ for a given temperature.

Bubble nucleation happens within a Hubble time if $S_{3} / T \lesssim 140$. In our scheme, because of the large prefactor $f^{3}$ in $S_{3}$, the nucleation starts when the Universe cools down close to $T=T_{2}$ so that the barrier of the potential is low enough. Here, $T_{2}$ is the temperature at which the barrier between minima of $V_{\text {eff }}$ disappears. At a temperature near $T_{2}$, the effective potential around $\theta=0$ can be approximated as

$$
\frac{2 V_{\text {eff }}}{\Lambda^{4}}= \begin{cases}\theta^{2}+\mathcal{O}\left(\theta^{4}\right) & \text { for } \theta>-\theta_{*} \\ \theta^{2}-\kappa^{2}\left(\theta+\theta_{*}\right)^{2}+\mathcal{O}\left(\theta^{3}\right) & \text { for } \theta<-\theta_{*}\end{cases}
$$

for $\kappa$ and $\theta_{*}$ depending on $T$ and the model parameters. Here, $\kappa$ is larger than unity, and $\theta_{*}$ is small and proportional to $\left(T / T_{2}-1\right) \sin \alpha$. It then follows that the curvature of the potential changes sign at $\theta=-\theta_{*}$. For $T>T_{2}$, one also finds that $V_{\text {eff }}=0$ at $\theta=0$ and $\theta=-\kappa \theta_{*} /(\kappa-1)$, and there is a potential barrier lying between the two points.

Let us now examine the bounce solution, which relies on the potential shape between the two points giving $V_{\text {eff }}=0$ at $T>T_{2}$. The equation of motion can be solved analytically because $\partial_{h} V_{\text {eff }}$ is approximately linear in $\theta$ in the relevant region. Introducing a dimensionless variable for simplicity

$$
\rho \equiv \sqrt{\kappa^{2}-1} \Lambda^{2} x
$$

we find that the solution is written 


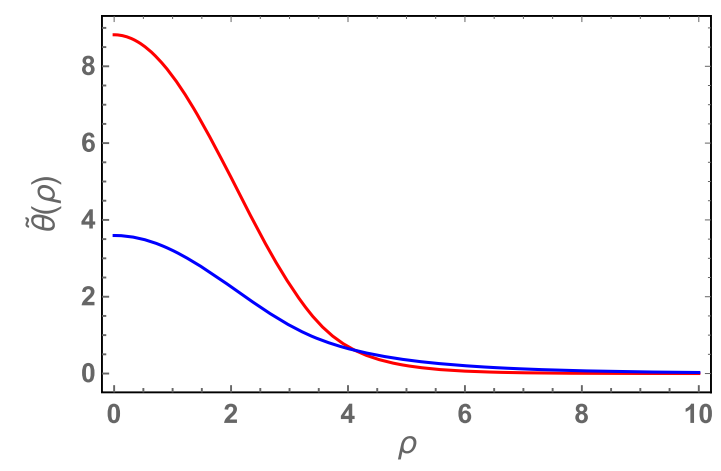

FIG. 5. Bounce solution for $\kappa=\sqrt{2}$ (red) and $2 \sqrt{2}$ (blue).

$$
\tilde{\theta} \equiv \frac{\theta}{\theta_{*}} \simeq \begin{cases}\frac{\kappa^{2}}{\kappa^{2}-1}+c_{1} \frac{\sin \rho}{\rho} & \text { for } \rho<\rho_{0} \\ 1 & \text { for } \rho=\rho_{0} \\ c_{2} \frac{\exp \left(-\rho / \sqrt{\kappa^{2}-1}\right)}{\rho} & \text { for } \rho>\rho_{0}\end{cases}
$$

where the coefficients $c_{1}$ and $c_{2}$ are given by

$$
\begin{aligned}
c_{1} & =\frac{\kappa^{2}\left(\rho_{0}+\sqrt{\kappa^{2}-1}\right)}{\left(\kappa^{2}-1\right)\left(\sqrt{\kappa^{2}-1} \cos \rho_{0}+\sin \rho_{0}\right)}, \\
c_{2} & =\frac{\kappa^{2} \exp \left(\frac{\rho_{0}}{\sqrt{\kappa^{2}-1}}\right)\left(\rho_{0} \cos \rho_{0}-\sin \rho_{0}\right)}{\left(\kappa^{2}-1\right) \cos \rho_{0}+\sqrt{\kappa^{2}-1} \sin \rho_{0}},
\end{aligned}
$$

which follows from the fact that $\theta$ and its derivative are continuous at $\rho=\rho_{0}$ with $\rho_{0}$ fixed by the condition

$$
\frac{\kappa^{2}\left(\rho_{0} \cos \rho_{0}-\sin \rho_{0}\right)}{\left(\kappa^{2}-1\right) \rho_{0} \cos \rho_{0}+\sqrt{\kappa^{2}-1} \rho_{0} \sin \rho_{0}}=1 .
$$

Note that $\rho_{0}$ is about $1.43 \pi$ in the limit $\kappa \rightarrow 1$, and it monotonically decreases with $\kappa$ while approaching to $\pi$. For instance, $\rho_{0} \simeq 1.18 \pi$ at $\kappa=\sqrt{2}$.

Figure 5 illustrates the profile of $\tilde{\theta}$ as a function of $\rho$. As one can see in the figure, the critical bubble has a thick wall because the field varies smoothly within the bubble of radius

$$
R_{c}=\frac{f}{\sqrt{\kappa^{2}-1} \Lambda^{2}} \rho_{0} \sim \frac{\pi}{\sqrt{\kappa^{2}-1}} \frac{f}{\Lambda^{2}} .
$$

The radius of the critical bubble in the real space can be regarded as the bubble wall width $L_{w}$ in the rest frame of the expanding bubble wall, i.e., $L_{w} \sim R_{c}$.

Finally, using the results obtained so far, one can estimate the bounce exponent around $T=T_{2}$ to be

$$
\frac{S_{3}}{T} \simeq c(\kappa) \frac{4 \pi f^{3} \theta_{*}^{2}}{T_{2} \Lambda^{2}}
$$

where $c(\kappa)$ is numerically calculable for a given $\kappa$, which is typically of order unity. For instance, $c(\sqrt{2}) \simeq 23$, $c(2 \sqrt{2}) \simeq 1.5$ and $c(7) \simeq 0.33$. It is important to notify that $S_{3} / T$ is proportional to $\left(T-T_{2}\right)^{2} f^{3}$ because $\theta_{*} \propto\left(T / T_{2}-1\right)$. Thus, in Eq. (19), $n$ is equal to 2 .

\section{APPENDIX B: RESIDUAL FIELD OSCILLATIONS INSIDE A BUBBLE}

The EW vacuum $(\phi, h)=\left(\phi_{T}, v_{T}\right)$ at $T$, which is determined by $\partial_{\phi} V=\partial_{h} V=0$, has

$$
-\alpha f \lesssim \phi_{T}<0, \quad 0<v_{T} \lesssim \Lambda
$$

and becomes the true vacuum when $T$ drops down below the critical temperature. Just after a critical bubble is formed at the nucleation temperature $T=T_{n}$, the Higgs and ALP fields take values

$$
\phi\left(t_{n}\right) \sim \theta_{*}\left(t_{n}\right) f \quad \text { and } \quad h\left(t_{n}\right) \ll v_{T},
$$

inside the bubble, and they are initially located far from the true vacuum. Here, we have used the fact that $\theta_{*}\left(t_{n}\right)$ is much smaller than $\alpha$ in size because $\theta_{*}$ is proportional to $\left(T / T_{2}-1\right) \sin \alpha$ and $T_{n}$ is close to $T_{2}$. Therefore, as the bubble expands, the fields classically roll toward the true vacuum while oscillating about it. Their evolution can be understood by looking deep inside the bubble, where the effect of spatial gradients are small. For a timescale much shorter than the Hubble time, the field evolution can be approximated by

$$
\begin{gathered}
\ddot{h}+\Upsilon_{h} \dot{h}+\partial_{h} V=0, \\
\ddot{\phi}+\Upsilon_{\phi} \dot{\phi}+\partial_{\phi} V=0,
\end{gathered}
$$

where the thermal dissipation rate $\Upsilon_{h}$ is determined by Higgs interactions to the background thermal plasma especially from top quark contribution [27],

$$
\Upsilon_{h} \sim\left\{\begin{array}{ll}
y_{t}^{2} \Gamma_{\mathrm{th}} & \text { for } y_{t} h \lesssim \Gamma_{\mathrm{th}} \\
y_{t}^{2} \frac{\left(y_{t} h\right)^{2}}{\Gamma_{\mathrm{th}}} & \text { for } y_{t} h \gtrsim \Gamma_{\mathrm{th}}
\end{array},\right.
$$

which includes an uncertainty of order unity due to the complicated dispersion relation for the top quark [26]. Note also that the weak gauge bosons give important contributions when the Higgs background field value $h$ is sizable. On the other hand, the anomalous coupling in Eq. (2) acts as a friction term, dissipating the ALP energy nonperturbatively with $[18,39,40]$

$$
\Upsilon_{\phi}=\frac{\Gamma_{\mathrm{sph}}}{T f^{2}} \sim 10^{-6} \frac{T^{3}}{f^{2}},
$$

for $T>E_{\mathrm{sph}}$, and

$$
\Upsilon_{\phi} \sim 10^{-5} \frac{T^{3}}{f^{2}}\left(\frac{2 m_{W}}{\alpha_{W} T}\right)^{7} e^{-\frac{E_{\mathrm{sph}}}{T}},
$$

for $T<E_{\mathrm{sph}}$, where the mass of the weak gauge boson and the sphaleron energy depend on the Higgs background field 
value as $m_{W}=g h / 2$ and $E_{\mathrm{sph}} \simeq 4 \pi h / g$, respectively. The trilinear ALP-Higgs-Higgs coupling can also give a sizable contribution to $\Upsilon_{\phi}$ during the period when the Higgs boson mass is comparable to or smaller than the temperature.

Subject to large thermal friction, the Higgs field is expected to be quickly frozen to the vacuum value $v_{T}$ within a timescale $1 / \Upsilon_{h} \sim 1 / T$. However, there is a residual oscillation induced by its mixing with the ALP, which is sizable even for a tiny mixing because the ALP has a large field excursion during its evolution. To examine qualitatively the field evolution, we take a quadratic approximation of the potential around the true vacuum

$$
V \simeq \frac{m_{L}^{2}}{2} \varphi_{L}^{2}+\frac{m_{H}^{2}}{2} \varphi_{H}^{2},
$$

for the light and heavy mass eigenstates given by

$$
\begin{gathered}
\varphi_{L}=\Delta \phi \cos \delta+\Delta h \sin \delta, \\
\varphi_{R}=\Delta h \cos \delta-\Delta \phi \sin \delta,
\end{gathered}
$$

which respectively have masses $m_{L} \simeq m_{\phi} \sim \Lambda^{2} / f$ and $m_{H} \simeq m_{h} \sim \Lambda$ at a given temperature. Here, $\Delta h \equiv h-v_{T}$ and $\Delta \phi \equiv \phi-\phi_{T}$ are the displacements from the true vacuum, and the mixing angle is roughly given by $\sin \delta \sim m_{\phi} / m_{h}$. It is straightforward to obtain the equations of motion in the canonical basis

$$
\begin{aligned}
\left(\frac{d^{2}}{d t^{2}}+\Upsilon_{h} \sin ^{2} \delta \frac{d}{d t}+m_{L}^{2}\right) \varphi_{L} & \simeq-\frac{\sin 2 \delta}{2} \Upsilon_{h} \dot{\varphi}_{H}, \\
\left(\frac{d^{2}}{d t^{2}}+\Upsilon_{h} \cos ^{2} \delta \frac{d}{d t}+m_{H}^{2}\right) \varphi_{H} & \simeq-\frac{\sin 2 \delta}{2} \Upsilon_{h} \dot{\varphi}_{L},
\end{aligned}
$$

with the initial conditions

$$
\begin{aligned}
& \varphi_{L}\left(t_{i}\right) \simeq-\phi_{T} \cos \delta-v_{T} \sin \delta \sim f, \\
& \varphi_{H}\left(t_{i}\right) \simeq-v_{T} \cos \delta+\phi_{T} \sin \delta \sim-\Lambda .
\end{aligned}
$$

Here, we have ignored the effect of the anomalous coupling on the motion of $\varphi_{L}$ because $\Upsilon_{\phi}$, which is smaller than about $10^{-6} T^{3} / f^{2}$, is much suppressed compared to the mixing-induced friction term $\Upsilon_{h} \sin ^{2} \delta \sim T v^{2} / f^{2}$ in the whole range of $h$ we are interested in. In the equation of motion for the heavy field $\varphi_{H}$, the term proportional to $\dot{\varphi}_{L}$ is much smaller than $\Lambda^{3}$ in size and so can be neglected until $\varphi_{H}$ gets close to its vacuum value. The thermal friction term thus quickly freezes $\varphi_{H}$ to the vacuum value within a timescale $1 / T$. For a timescale much shorter than the Hubble time, the solutions are approximated to be

$$
\begin{aligned}
& \varphi_{L} \approx \phi_{T} e^{-\delta^{2} \Upsilon_{h} t / 2} \cos \left(\sqrt{m_{\phi}^{2}-\delta^{4} \Upsilon_{h}^{2}} t+\beta_{L}\right), \\
& \varphi_{H} \approx v_{T} e^{-\Upsilon_{h} t / 2} \cos \left(\sqrt{m_{h}^{2}-\Upsilon_{h}^{2}} t+\beta_{H}\right),
\end{aligned}
$$

at temperatures below $T_{n}$, where $\beta_{L}$ and $\beta_{H}$ are constant phases. We have numerically confirmed the above approximations. Note also that the Higgs field evolves according to

$$
h(t) \approx v_{T}-\phi_{T} \sin \delta \times e^{-\delta^{2} \Upsilon_{h} t / 2} \cos \left(m_{\phi} t+\beta_{L}\right),
$$

which follows from $\Delta h \simeq \varphi_{L} \sin \delta$ for $t \gg 1 / T$. Because $\phi_{T} \sin \delta \sim \Lambda$, the residual oscillation of the Higgs field can be sizable for a timescale less than about $1 /\left(\delta^{2} T\right)$. Figure 2, which is obtained via a numerical calculation, illustrates such a feature.

The results above can be understood more easily by replacing the heavy Higgs field $h$ with $\hat{h}(\phi)$ because the rapid damping $\varphi_{H} \rightarrow 0$ means $\partial V / \partial h \rightarrow 0$ for a given value of $\phi$. In such constructed effective theory, the ALP oscillates about the true minimum $\phi=\phi_{T}$ after tunneling, and accordingly, the Higgs background field value changes because it is given by $v(t)=\hat{h}(\phi(t))$. Note also that the ALP effectively couples to SM particles with a coupling proportional to $\partial \hat{h} /\left.\partial \phi\right|_{\phi_{T}}$, and thus its oscillating energy is thermally dissipated.

For a final remark, we note that the real situation is more complicated because the mixing angle is field dependent, and self-interactions during bubble expansion are also important. Our point here is that there can be sizable Higgs oscillations, which would then wash out the baryon asymmetry. In the analysis, we have taken into account such effects to obtain a conservative estimation of the final baryon abundance.
[1] V. Andreev et al. (ACME Collaboration), Nature (London) 562, 355 (2018).

[2] L. Bian, T. Liu, and J. Shu, Phys. Rev. Lett. 115, 021801 (2015).
[3] J. M. Cline, K. Kainulainen, and D. Tucker-Smith, Phys. Rev. D 95, 115006 (2017).

[4] I. Baldes and G. Servant, J. High Energy Phys. 10 (2018) 053. 
[5] K. S. Jeong, T. H. Jung, and C. S. Shin, Phys. Lett. B 790, 326 (2019).

[6] J. Jaeckel and M. Spannowsky, Phys. Lett. B 753, 482 (2016).

[7] S. Knapen, T. Lin, H. K. Lou, and T. Melia, Phys. Rev. Lett. 118, 171801 (2017).

[8] A. Mariotti, D. Redigolo, F. Sala, and K. Tobioka, Phys. Lett. B 783, 13 (2018).

[9] X. C. Vidal, A. Mariotti, D. Redigolo, F. Sala, and K. Tobioka, J. High Energy Phys. 01 (2019) 113.

[10] A. I. Bochkarev and M. E. Shaposhnikov, Mod. Phys. Lett. A 02, 417 (1987).

[11] J. M. Cline, M. Joyce, and K. Kainulainen, J. High Energy Phys. 07 (2000) 018.

[12] A. G. Cohen, D. B. Kaplan, and A. E. Nelson, Phys. Lett. B 263, 86 (1991).

[13] G. F. Giudice and M. E. Shaposhnikov, Phys. Lett. B 326, 118 (1994).

[14] M. Dine and S. D. Thomas, Phys. Lett. B 328, 73 (1994).

[15] M. Joyce, T. Prokopec, and N. Turok, Phys. Lett. B 339, 312 (1994).

[16] A. G. Cohen, D. B. Kaplan, and A. E. Nelson, Phys. Lett. B 336, 41 (1994).

[17] M. Joyce, T. Prokopec, and N. Turok, Phys. Rev. Lett. 75, 1695 (1995); 75, 3375(E) (1995).

[18] M. D’Onofrio, K. Rummukainen, and A. Tranberg, Phys. Rev. Lett. 113, 141602 (2014).

[19] M. E. Shaposhnikov, Nucl. Phys. B299, 797 (1988).

[20] M. Dine, P. Huet, R. L. Singleton, Jr., and L. Susskind, Phys. Lett. B 257, 351 (1991).

[21] J. Garcia-Bellido, D. Y. Grigoriev, A. Kusenko, and M. E. Shaposhnikov, Phys. Rev. D 60, 123504 (1999).

[22] T. Konstandin and G. Servant, J. Cosmol. Astropart. Phys. 07 (2011) 024.
[23] G. Servant, Phys. Rev. Lett. 113, 171803 (2014).

[24] N. Craig and J. March-Russell, arXiv:1007.0019.

[25] P. W. Graham, D. E. Kaplan, and S. Rajendran, Phys. Rev. Lett. 115, 221801 (2015).

[26] S. Y. Wang, D. Boyanovsky, H. J. de Vega, D. S. Lee, and Y. J. Ng, Phys. Rev. D 61, 065004 (2000).

[27] K. Mukaida and K. Nakayama, J. Cosmol. Astropart. Phys. 01 (2013) 017.

[28] K. Choi and S. H. Im, J. High Energy Phys. 12 (2016) 093.

[29] T. Flacke, C. Frugiuele, E. Fuchs, R. S. Gupta, and G. Perez, J. High Energy Phys. 06 (2017) 050.

[30] R. Aaij et al. (LHCb Collaboration), J. High Energy Phys. 02 (2013) 105.

[31] J.-T. Wei et al. (Belle Collaboration), Phys. Rev. Lett. 103, 171801 (2009).

[32] R. Aaij et al. (LHCb Collaboration), Phys. Rev. Lett. 115, 161802 (2015).

[33] F. Bezrukov and D. Gorbunov, Phys. Lett. B 736, 494 (2014).

[34] H. J. Hyun et al. (Belle Collaboration), Phys. Rev. Lett. 105, 091801 (2010).

[35] S. Adler et al. (E787 Collaboration), Phys. Rev. D 70, 037102 (2004).

[36] A. V. Artamonov et al. (BNL-E949 Collaboration), Phys. Rev. D 79, 092004 (2009).

[37] F. Bergsma et al. (CHARM Collaboration), Phys. Lett. B 157, 458 (1985).

[38] S. Alekhin et al., Rep. Prog. Phys. 79, 124201 (2016).

[39] L. D. McLerran, E. Mottola, and M. E. Shaposhnikov, Phys. Rev. D 43, 2027 (1991).

[40] Y. Burnier, M. Laine, and M. Shaposhnikov, J. Cosmol. Astropart. Phys. 02 (2006) 007. 